\title{
APPROXIMATING FIXED POINTS OF SOME MAPPINGS
}

\author{
RAMENDRA KRISHNA BOSE AND RATHINDRA NATH MUKHERJEE
}

\begin{abstract}
In a uniformly convex Banach space, Senter and Dotson, Jr., have given conditions under which certain types of iterates of a quasi-nonexpansive mapping converge to a fixed point of the mapping. Here we consider two types of mappings, one considered by Ray and the other considered by Goebel, Kirk and Shimi, and prove some results concerning the approximations of fixed points of such mappings. A result of Kannan is obtained as a particular case of our result under relaxed conditions.
\end{abstract}

Introduction. Dotson, Jr., introduced in [1] a new class of mappings called quasi-nonexpansive mappings. Let $X$ be a Banach space and let $C$ be a convex subset of $X$. A self-mapping $T$ of $C$ is said to be quasi-nonexpansive provided $T$ has a fixed point in $C$ and if $p \in C$ is a fixed point of $T$, then

$$
\|T(x)-p\| \leqslant\|x-p\|
$$

is true for all $x \in C$.

In a uniformly convex Banach space, Senter and Dotson, Jr. [6] have given conditions under which certain types of iterates (Mann type [4]) of a quasi-nonexpansive mapping converge to a fixed point of the mapping. In the present work, we consider two particular types of mappings, one type considered by Ray [5] and the other type considered by Goebel, Kirk and Shimi [2], and prove some results concerning the approximations of fixed points of such mappings. A result proved by Kannan [3] is obtained as a particular case of our result under relaxed conditions.

1. Ray [5] proved the following theorem.

THEOREM 1. Let $C$ be a nonempty, bounded, closed and convex subset of a reflexive Banach space $X$ and let $C$ have a normal structure. If $T$ is a mapping from $C$ into itself, such that

$$
\begin{gathered}
\|T(x)-T(y)\|<a\|x-y\|+b\{\|x-T(x)\|+\|y-T(y)\|\} \\
+c\{\|y-T(x)\|+\|x-T(y)\|\}
\end{gathered}
$$

for all $x, y \in C$ where $a, b, c>0$ and $3 a+2 b+4 c<1$, then $T$ has $a$ unique fixed point.

Goebel, Kirk and Shimi [2] proved the following theorem.

Received by the editors May 21, 1980 and, in revised form, August 29, 1980.

AMS (MOS) subject classifications (1970). Primary 47H10; Secondary 47H99.

(c) 1981 American Mathematical Society 0002-9939/81/0000-0368/\$02.00 
TheOREM 2. Let $X$ be a uniformly convex Banach space, $C$ a nonempty bounded, closed and convex subset of $X$ and $T: C \rightarrow C$ a continuous mapping such that

$$
\begin{gathered}
\|T(x)-T(y)\|<a\|x-y\|+b\{\|x-T(x)\|+\|y-T(y)\|\} \\
+c\{\|x-T(y)\|+\|y-T(x)\|\}
\end{gathered}
$$

for all $x, y \in C$ where $a, b, c \geqslant 0$ and $a+2 b+2 c<1$. Then $T$ has $a$ fixed point in $C$.

Next we give certain conditions introduced by Senter and Dotson, Jr. [6]. A mapping $T: C \rightarrow C$ with a nonempty fixed point set $F(T)$ in $C$ is said to satisfy

Condition $I$. If there is a nondecreasing function $f:[0, \infty) \rightarrow[0, \infty)$ with $f(0)=0$, $f(r)>0$ for $r \in(0, \infty)$ such that $\|x-T(x)\|>f(d(x, F(T)))$ for all $x \in C$, where $d(x, F(T))=\inf \{\|x-z\|: z \in F(T)\}$.

Similarly the above mapping $T$ is said to satisfy

Condition II. If there is a real number $\alpha>0$ such that $\|x-T(x)\|>$ $\alpha d(x, F(T))$ holds for all $x \in C$.

It can be easily seen that a mapping which satisfies Condition II also satisfies Condition I. Now we state a key theorem of Senter and Dotson, Jr., [6] which we use in our work. Let $P$ denote the set of positive integers. Let $x_{1} \in C$ and let $M\left(x_{1}, t_{n}, T\right)$ be the sequence $\left\{x_{n}\right\}$ defined by $x_{n+1}=\left(1-t_{n}\right) x_{n}+t_{n} T\left(x_{n}\right)$ where $t_{n} \in[\beta, \gamma], 0<\beta<\gamma<1$ and $n \in P$.

Theorem 3. Suppose $X$ is a uniformly convex Banach space, $C$ is a closed, convex subset of $X$ and $T$ is a quasi-nonexpansive mapping of $C$ into $C$. If $T$ satisfies Condition I, then for arbitrary $x_{1} \in C, M\left(x_{1}, t_{n}, T\right)$ converges to a member of $F(T)$.

Now we state our theorems in this direction.

THEOREM 4. Let $X$ be a uniformly convex Banach space. Let $C$ be a nonempty closed, convex and bounded subset of $X$. If $T$ is a mapping from $C$ into itself such that

$$
\begin{gathered}
\|T(x)-T(y)\| \leqslant a\|x-y\|+b\{\|x-T(x)\|+\|y-T(y)\|\} \\
+c\{\|x-T(y)\|+\|y-T(x)\|\}
\end{gathered}
$$

for all $x, y \in C$ where $a, b, c>0$ and $3 a+2 b+4 c<1$, then for arbitrary $x_{1} \in$ $C, M\left(x_{1}, t_{n}, T\right)$ converges to the unique fixed point of $T$.

Proof. By Theorem 1, the mapping $T$ has a unique fixed point (as $C$ has a normal structure). Also $T$ satisfies the condition $\|T(x)-p\|<\|x-p\|, \forall x \in C$ where $p$ is the fixed point of $T$. For

$$
\begin{aligned}
\|T(x)-p\| & =\|T(x)-T(p)\| \\
& \leqslant(a+c)\|x-p\|+b\|T(x)-x\|+c\|T(x)-p\| \\
\text { i.e., }(1-b-c) \| T(x) & -p\|<(a+b+c)\| x-p \| \text { i.e., } \\
\|T(x)-p\| & \leqslant \frac{a+b+c}{1-b-c}\|x-p\|<\frac{1-2 a-b-3 c}{1-b-c}\|x-p\| \\
& =\left\{1-\frac{2 a+2 c}{1-b-c}\right\}\|x-p\|
\end{aligned}
$$


i.e., $\|T(x)-p\|<\{1-(2 a+2 c) /(1-b-c)\}\|x-p\|<\|x-p\|$. Hence $T$ is quasi-nonexpansive.

Also we have

$$
\begin{aligned}
\|T(x)-p\| & <(a+c)\|x-p\|+b\|T(x)-x\|+c\|T(x)-p\| \\
& <(a+2 c)\|x-p\|+(b+c)\|T(x)-x\| .
\end{aligned}
$$

Also we know that $\|T(x)-p\| \geqslant\|x-p\|-\|x-T(x)\|$. Therefore

$$
(b+c)\|T(x)-x\|+(a+2 c)\|x-p\|>\|x-p\|-\|x-T(x)\|
$$

i.e.,

$$
\|x-T(x)\|>(1-a-2 c / 1+b+c)\|x-p\|=\alpha\|x-p\|,
$$

where $\alpha=(1-a-2 c) /(1+b+c)>0$. Hence $T$ satisfies Condition II (hence Condition I). So by Theorem 3 , the result follows.

We get a result proved by Kannan [3] under relaxed conditions as a particular case of the above theorem. Kannan [3] proved the following theorem.

THEOREM 5. Let $C$ be a nonempty bounded, closed and convex subset of a uniformly convex Banach space $X$. Let $T$ be a mapping of $C$ into itself such that

(i) $\|T(x)-T(y)\| \leqslant \frac{1}{2}\|x-T(x)\|+\frac{1}{2}\|y-T(y)\|, x, y \in C$, and

(ii) $\sup _{z \in K}\|\mathrm{z}-T(z)\| \leqslant \delta(K) / 2$, where $K$ is any nonempty convex subset of $C$ 'which is mapped into itself by $T(\delta(K)$ is the diameter of $K)$.

Then the sequence $\left\{x_{n}\right\}$ where $x_{n+1}=\frac{1}{2} x_{n}+\frac{1}{2} T\left(x_{n}\right)$, converges to the fixed point of $T$ in $C$, where $x_{1}$ is any arbitrary point of $C$.

In Theorem 4, take $a=c=0, b=\frac{1}{2}$ and $t_{n}=\frac{1}{2}, n \in P$. This proves the assertion of the above theorem of Kannan [3] without requiring condition (ii).

THEOREM 6. Let $C$ be a nonempty bounded, closed and convex subset of a uniformly convex Banach space $X$. Suppose $T$ is a continuous mapping of $C$ into itself such that

$$
\begin{gathered}
\|T(x)-T(y)\| \leqslant a\|x-y\|+b\{\|x-T(x)\|+\|y-T(y)\|\} \\
+c\{\|x-T(y)\|+\|y-T(x)\|\},
\end{gathered}
$$

$x, y \in C$, where $a+2 b+2 c \leqslant 1, a, c \geqslant 0$ and $b>0$. Then $M\left(x_{1}, t_{n}, T\right)$ converges to a fixed point of $T$.

Proof. By Theorem 2, $T$ has a fixed point in $C$. As in the proof of Theorem 4, it can be easily shown that $T$ is quasi-nonexpansive and $T$ satisfies Condition $I$. Hence by Theorem 3, $M\left(x_{1}, t_{n}, T\right)$ converges to a fixed point of $T$.

Remarks. We have come across a paper in this direction by Shimi (Approximation of fixed points of certain non-linear mappings, J. Math. Anal. Appl. 65 (1978), 565-571) but our results and proofs are different. Also we note that the theorem of Kannan [3] proved here under relaxed conditions cannot be obtained from results obtained by Shimi. 


\section{REFERENCES}

1. W. G. Dotson, Jr., Fixed points of quasi-nonexpansive mappings, J. Austral. Math. Soc. 13 (1972), 167-170.

2. K. Goebel, W. A. Kirk and T. N. Shimi, A fixed point theorem in uniformby convex spaces, Boll. Un. Mat. Ital. 7 (1973), 67-75.

3. R. Kannan, Some results on fixed points. III, Fund. Math. 70 (1971), 170-177.

4. W. R. Mann, Mean value methods in iteration, Proc. Amer. Math. Soc. 4 (1953), 506-510.

5. B. K. Ray, A fixed point theorem in Banach space, Indian J. Pure Appl. Math. 8 (1977), 903-907.

6. H. F. Senter and W. G. Dotson, Jr., Approximating fixed points of nonexpansive mappings, Proc. Amer. Math. Soc. 44 (1974), 375-379.

Department of Mathematics, Birla Institute of Technology and Science, Pilani, Rajasthan333 031, India (Current address of R. K. Bose)

Current address (R. N. Mukherjee): Department of Mathematics, Institute of Technology, Banaras Hindu University, Varanasi, India 